\title{
Proceso de Pruebas de Usabilidad de Software
} Software Usability Testing Process

\author{
Sánchez Morales G.', Mezura-Godoy C.ㄹ, Benítez-GuerreroE. ${ }^{3}$ \\ Facultad de Estadística e Informática, CONACYT-Universidad Veracruzana, \\ Av. Xalapa, S/N, 91020, Xalapa, Veracruz. México. \\ Facultad de Estadística e Informática, Universidad Veracruzana, \\ Av. Xalapa, S/N, 91020, Xalapa, Veracruz. México. \\ Facultad de Estadística e Informática, Universidad Veracruzana, \\ Av. Xalapa, S/N, 91020, Xalapa, Veracruz. México \\ ${ }^{1}$ gsanchezmo@conacyt.mx, ${ }^{2}$ cmezura@uv.mx, sedbenitez@uv.mx
}

Fecha de recepción: 15 de junio 2017

Fecha de aceptación: 2 de febrero 2018

Resumen. Existen diversos trabajos que orientan las pruebas de usabilidad los cuales son muy generales o demasiados específicos, ocasionando que las personas que empiezan a incursionar en el ámbito de las pruebas de usabilidad se sientan desorientados por el exceso de información. En consecuencia, en este trabajo se propone un proceso de pruebas de usabilidad de software, mostrando los elementos necesarios, incluyendo algunas sugerencias y recomendaciones que permitan incursionar a las pruebas de usabilidad de manera fácil.

Palabras Clave: Pruebas de Usabilidad, Proceso, Software.

Summary. There are several works that guide usability testing that are generally or too much specific; causing people who are beginners at usability testing feel confused by the excess information. Consequently, this paper presents a Software Usability Testing Process, this process shows the necessary elements, including some suggestions and recommendations that make it easy to enter usability testing.

Keywords: Usability testing, Process, Software.

\section{Introducción}

Una forma de definir si una aplicación de software es usable, es evaluando si cumple con características de usabilidad. La usabilidad se define como el alcance de un producto o servicio usado por usuarios específicos, para lograr un objetivo con efectividad, eficiencia y satisfacción en un contexto específico. La efectividad se valora en términos de la precisión y completitud con la que el usuario alcanza sus objetivos, así como los errores cometidos, mientras que la eficiencia se refiere al tiempo y esfuerzo para alcanzar los objetivos. La satisfacción se considera en términos de la ausencia de incomodidad y una actitud positiva hacia el uso de la aplicación. En cuanto al contexto, se refiere a los diferentes aspectos tales como: usuarios, tareas, equipo requerido por el software, hardware y materiales, así como el entorno físico y social en el cual un producto es usado [1].

Una manera de evaluar la usabilidad es mediante pruebas de usabilidad, las cuales involucran medir la ejecución de usuarios sobre la realización de ciertas tareas en un ambiente controlado, como lo es un laboratorio de usabilidad [2].

Las pruebas de usabilidad comprenden tres técnicas que en conjunto permiten medir la efectividad, eficiencia y satisfacción. La primera técnica se llama pruebas con usuarios, ésta prueba permite que cierto tipo de usuarios realicen tareas específicas para que a partir de sus ejecuciones se evalúe la usabilidad y se identifique errores. La segunda técnica es la observación que tiene como objetivo percibir las actividades de los usuarios mediante la grabación de sus ejecuciones (interacción con el sistema, registro de actividades), sus movimientos, gestos faciales y corporales; también se puede hacer dichas observaciones por una persona dedicada a esa actividad. Mediante la observación se pueden identificar tareas exitosas, es decir, aquellas completadas en el tiempo deseado o con tiempo extra, también se puede considerar las tareas exitosas con o sin ayuda; sin olvidarse de contabilizar las tareas sin éxito. De igual manera, se considera los errores cometidos por los usuarios de manera general, en determinado tiempo o clasificándolos de alguna manera. Incluso contabilizar la frecuencia en usar la ayuda. Debido a los datos que se pueden obtener a partir de la observación se pueden medir la efectividad y la eficiencia. La tercera técnica consiste en preguntar a los usuarios mediante el uso de cuestionarios y entrevistas sobre la opinión y satisfacción en el uso de la herramienta. Actualmente, existen diversos cuestionarios predefinidos que permiten conocer dicha satisfacción; por ejemplo, el cuestionario CSUQ (Computer System Usability Questionnaire) [3].

Es importante señalar que para llevar acabo las pruebas de usabilidad se debe seguir un proceso que determine las pautas necesarias. De cierta manera, algunos trabajos como [2, 4] proveen un proceso para hacer dichas pruebas siguiendo principalmente los siguientes puntos: i) establecer un objetivo; ii) realizar actividades relacionadas a los usuarios, a la aplicación a evaluar; iii) llevar a cabo la observación y las entrevistas; finalmente iv) analizar resultados. Otro trabajo engloba los proceso anteriores en las siguientes categorías: planeación, preparación, conducción, análisis y reporte [5].

En una u otra medida, cada uno de estos trabajos proporcionan detalles que orientan a los novatos en pruebas de usabilidad, pero se ha observado en la práctica que aún cuando existen estos procesos al momento de llevar a cabo dichas pruebas las personas novatas suelen confundirse con dicha información por ser muy extensa o 
demasiada breve. Por ello nos interesamos en las propuestas de evaluación de la usabilidad existentes, con el fin de proponer un proceso de pruebas de usabilidad para novatos o personas que se inician en el proceso de evaluación de software, que incluya formatos a usar, sugerencias y recomendaciones.

La distribución de este artículo es la siguiente: en la sección 2 se presenta algunos procesos para llevar a cabo la pruebas de usabilidad; en la sección 3 se muestra nuestra propuesta para hacer dichas pruebas, este proceso considera la planeación, la ejecución, el análisis y el reporte; en la sección 4 se muestra el resultado de poner a la práctica el proceso propuesto, mientras en la sección 5 se presentan las conclusiones y el trabajo a futuro.

\section{Estado del arte}

Preece [2] propone un proceso para hacer las pruebas de usabilidad considerando el marco DECIDE; que incluye 5 puntos. El primero es establecer el objetivo y explorar las preguntas que ayudaran a responder el objetivo; el segundo paso es determinar el paradigma y las técnicas, el paradigma corresponde a las pruebas con usuarios, mientras que las técnicas comprenden registrar datos mediante video y log de la interacción de los usuarios, cuestionarios de satisfacción y entrevista. El tercer paso es la identificación de puntos prácticos, que incluye: a) selección de las tareas para los usuarios, b) selección de usuarios, c) preparación del lugar para las prueba y d) planeación de la ejecución de las pruebas. En el inciso 'a)' se recomienda que cada tarea (entre 5 y $20 \mathrm{~min}$ ) debe probar un problema. Para el inciso 'b)', la selección de usuarios, se debe considerar la edad o la experiencia; se recomienda considerar las características de los usuarios finales sin hacer sesgos y equilibrar la participación de hombre y mujeres a menos que la aplicación a probar este orientada a cierto género. En el caso del inciso 'c)' se considera utilizar un laboratorio de usabilidad o preparar un espacio; incluso arreglar el área de la prueba con cierto parecido al lugar en donde se planea operar la aplicación. En el inciso 'd)' se consideran 4 diferentes scripts (saludo a los participantes, explicación de la prueba, explicación del comportamiento de los usuarios y una introducción a la tarea exploratoria) que ayude a conducir la prueba; también se recomienda hacer una prueba piloto para asegurar que todo está bien, incluso se debe considerar tener un plan de qué hacer cuando no se logre la tarea en el tiempo estimado. El punto cuarto concierne a la ética, lo que implica crear una hoja de consentimiento, indicar el uso de cámaras de grabación y si existe otro tipo de observación. El quinto punto se refiere a la evaluación, al análisis y a la presentación de los datos recabados; en esta parte se recomienda recabar la información a partir de la observación, usar estadística descriptiva como máximos, mínimos, promedio del grupo y desviación estándar para presentar la información.

Otro trabajo, [4], se identifican 7 elementos básicos a considerar en las pruebas de usabilidad: 1) desarrollar las preguntas de investigación o los objetivos de la prueba, 2) empleo de una muestra representativa de usuarios que pueden o no ser elegidos al azar, 3) representación del entorno actual de trabajo, 4) observación del usuario al usar o revisar el producto a evaluar, 5) entrevistas y pruebas de los usuarios, 6) colección de ejecuciones cualitativas, cuantitativas y mediciones de preferencias y 7) recomendaciones de mejoras para el producto.

En la fuente [5] recomienda hacer en 5 etapas las pruebas de usabilidad. La primera etapa, planeación, se establece el objetivo, aspectos que afectan las pruebas. En la etapa dos, preparación de la prueba; se proveen opciones para reclutar los usuarios, asignar roles y responsabilidades (y hacer un checklist para cada rol que considera antes, durante y después de la prueba), hacer diversos formatos (de consentimiento para grabación y menores de edad, acerca confidencialidad, notas de los observadores), crear cuestionarios (antes de la prueba para recabar información del usuario, después de cada tarea y al finalizar la prueba para conocer la experiencia) o buscar cuestionarios que sean usados al finalizar la prueba. La etapa tres, conducir la prueba, considera preparar el lugar, pensar en las preguntas antes de la prueba, sugerencias de cómo hacer las pruebas solo o acompañado con dos o más participantes; provee sugerencias de qué hacer al presentarse algunos problemas, así como de conducir a los observadores y visitantes. En la etapa cuatro, análisis de lo encontrado, permite analizar la información de los observadores, los videos, los registros de interacción y los gestos. Mencionan la ventaja y desventajas de usar un conjunto de heurísticas; de manera similar provee la opción de agrupar las observaciones sin seguir una heurística. Se recomienda identificar los datos cualitativos (tiempo al completar una tarea, errores cometidos, tareas sin concluir, asistencias ) y cuantitativos (usando la media, moda y promedio) y finalmente priorizar el orden de problemas. La etapa cinco, preparar un reporte, considera si los resultados serán por escrito (reporte o presentación) u oral, en la parte reporte involucra el reporte formal e informal. Dentro del reporte formal considera puntos que van desde una portada, versión de la aplicación evaluada, lugar usado, el perfil de los participantes usuarios, los objetivos, resultados después de cada tarea y al finalizar la prueba, recomendaciones, trabajo a futuro y apéndices.

Dado el análisis anterior, se observa que algunos trabajos pueden llegar a confundir a los evaluadores novatos debido a que estos trabajos son muy detallados o muy generales; por lo anterior, en este trabajo se muestran los elementos necesarios, incluyendo algunas sugerencias y recomendaciones que permitan incursionar a las pruebas de usabilidad de manera fácil.

\section{Proceso de pruebas de usabilidad}

Como se observa en la Figura 1 se propone 3 etapas para el proceso de pruebas de usabilidad que incluye: i) planeación, es decir, desde la definición de los objetivos hasta la realización de formatos y cuestionarios, ii) 
ejecución, en donde se proveen consejos de cómo llevar a acabo la prueba, y finalmente iii) analizar y reportar sobre los datos recopilados de las prueba de usabilidad.

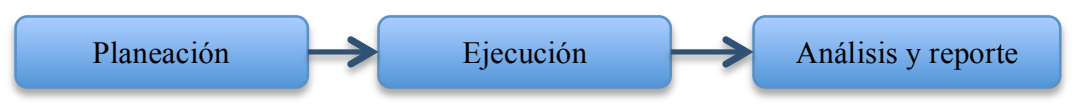

Figura 1. Proceso de las pruebas de usabilidad.

\subsection{Planeación}

En la planeación de las pruebas de usabilidad se considerar 4 componentes: 1) objetivos y preguntas de apoyo, 2) tareas, 3 ) recursos y 4) plan de ejecución. Es recomendable realizar formatos desde el punto 2 con la finalidad de tener una mejor organización (ver Figura 2).

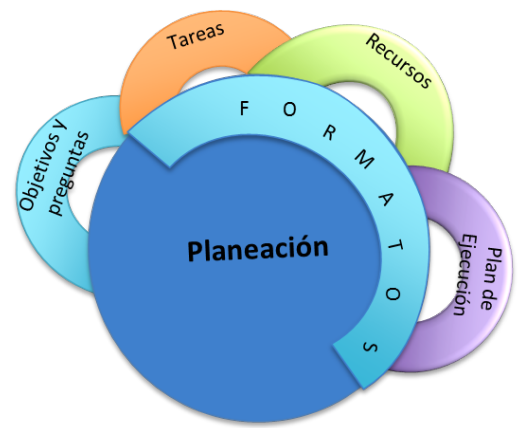

Figura 2. Componentes de la planeación.

1) Objetivos y preguntas de apoyo. Definir los objetivos de la evaluación, los cuales frecuentemente responden a qué tan usable es la aplicación; mientras que las preguntas de apoyo ayudan a resolver dichos objetivos; por ejemplo, ¿podrán los usuarios registrarse en 2 minutos? o ¿podrán los usuarios realizar la compra de un producto sin problemas?.

2) Tareas. Consiste en i) establecer las tareas que realizarán los usuarios para evaluar la aplicación y ii) establecer el tiempo de cada tarea. Para el punto i se considera que las tareas se relacionan con las preguntas de apoyo o con las funcionalidades representativas de la aplicación; por ejemplo, comprar un objeto que ofrece la aplicación o registrarse dentro del sistema. El punto $i i$ se puede lograr considerando experiencias previas o solicitando a alguien, que no pertenezca al equipo de desarrollo, en la realización de dichas tareas tomando en cuenta su experiencia en el uso de dichas aplicaciones. En este último punto sirve para: 1) poner tiempo límite para realizar las tareas, ya que al llevar a acabo estas, el usuario puede encontrar dificultades e invertir mucho tiempo en resolverla, generando estrés y 2) reportar las tareas que fueron realizadas en el tiempo estimado.

Sugerencia y/o recomendación: Realizar formato que enliste las tareas a realizar por los usuarios; se sugiere agregar un escenario, preguntas relacionadas con la satisfacción o sugerencias, como se ve en la Figura 3. Este formato puede ser entregado a los usuarios o ser leído por el moderador al inicio de cada tarea. Otro formato puede ser para los observadores, usando las tareas seleccionadas; en este se sugiere solicitar que los observadores agreguen el tiempo empleado por cada tarea desempeñada por el usuario, errores cometidos, las diferentes emociones, las distracciones, etc.

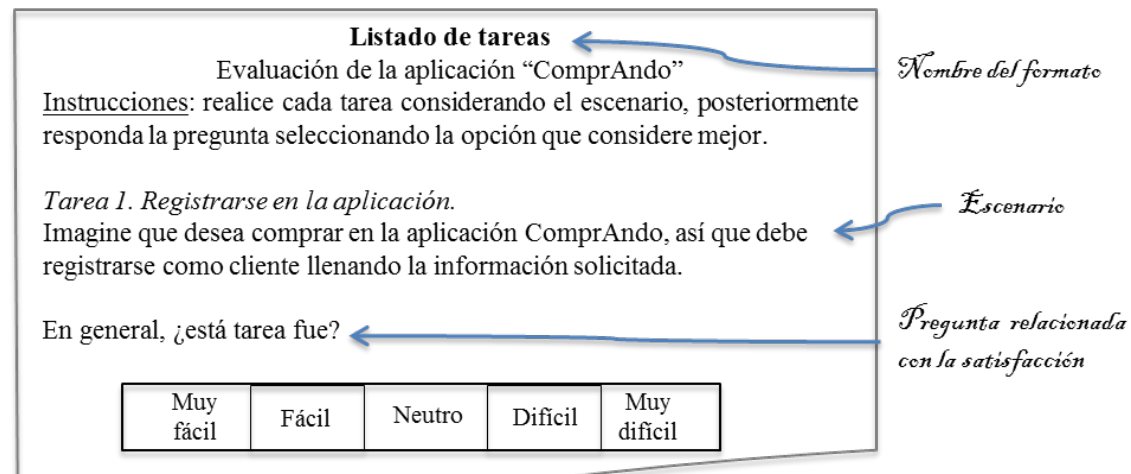

Figura 3. Formato de tareas solicitadas a los usuarios. 
3) Recursos. Involucra i) recursos humanos (RH), ii) recursos físicos y iii) recursos de evaluación. i) Los RH incluye los usuarios y el equipo de pruebas. En el caso de los usuarios se requiere conocer la cantidad de ellos (de 3 a 5 usuarios [6]) para la pruebas, además que cubran el perfil requerido. El perfil considera el nivel de experiencia, rango de edades, género, preferencias, habilidades o capacidades diferentes, etc., es decir, aspectos relevantes para la aplicación.

Recomendación y sugerencias: los usuarios no deben ser parte del equipo de desarrollo y se debe equilibrar la cantidad de hombres y mujeres, incluso se debe considera cómo se reclutará a los usuarios, si se les pagará o se les ofrecerá algo de comer o beber. Se recomienda hacer un formato de consentimiento en el caso de usuarios menores de edad o cuando se grabe al usuario.

Respecto al recurso de evaluación o equipo de las pruebas se refiere a los roles requeridos que pueden ser cubiertos por una sola persona o por varias dependiendo de la disponibilidad de personas; por tal razón se hará mención respecto a los roles (Moderador, Observador y Técnico). El Moderador es quien conducirá la prueba, dará la bienvenida a los participantes, leerá y entregará los formatos generados, entre otras cosas. El Observador observa las ejecuciones de las tareas por parte de los participantes, aspectos como el tiempo empleado en cada tarea, las dificultades encontradas y el estado de ánimo. El Técnico verificará las instalaciones requeridas en la prueba, la colocación de cámaras, la instalación de la aplicación, la conexión a internet, así como otras necesidades técnicas.

ii) Los recursos físicos considera el hardware, el software y el lugar para realizar las pruebas. El hardware considera el o los dispositivos en donde se ejecutará la aplicación a evaluar, las cámaras de video en caso de hacer grabaciones, etc.; mientras que el software se refiere a la aplicación a evaluar y/o las herramientas de grabación (interacciones y el rostro del usuario). El lugar para realizar las pruebas es en laboratorios de usabilidad.

Sugerencia y/o recomendación: Se sugiere hacer un formato tipo checklist para asegurarse que el día de la prueba se ha realizado todo lo necesario respecto a los recursos físico. Si no cuentas con laboratorio de usabilidad acondiciona un cuarto considerando lo siguiente: un lugar fresco, iluminación adecuada, espacioso para el usuario y el equipo de evaluación, con conexiones a la toma corriente, acceso a internet, poco ruidoso e instalación de cámara si es necesario.

iii) Recursos de evaluación se refiere a seleccionar la herramienta de evaluación que ayudará a evaluar la satisfacción o de manera general la usabilidad. Existen cuestionarios par evaluar la satisfacción, por ejemplo: CSUQ versión en español [7], QUIS (The Questionnaire for User Interaction Satisfaction) [8] y SUMMI (Software Usability Measurement Inventory) [9]. Para medir la usabilidad de manera general esta el cuestionario SUS [10] y SIRIUS. Recomendación: determinar si el cuestionario será contestado en papel o en computadora.

4) Planeación de ejecución. Se considera la realización 2 actividades:

i) Redactar un guión de ejecución con los siguientes puntos: agradecimiento, explicación de la aplicación a evaluar, objetivo de la evaluación, actividades a realizar, explicación si serán observados, y

ii) Realizar el programa de actividades de acuerdo al guión.

Sugerencia y/o recomendación:

1. Hacer un formato para el guión y establecer el tiempo por cada actividad del guión; si las realización de las tareas duran más de 25 minutos se debe tener un descanso.

2. Hacer un formato tipo checklist que considere actividades a realizar antes, durante y después de la prueba, como se observa en la Tabla 1.

Recomendación: Aclarar a los usuarios que se está probando la aplicación no sus conocimientos.

Tabla 1. Formatos sugeridos para el plan de ejecución.

\begin{tabular}{lll}
\hline $\begin{array}{l}\text { Nombre del } \\
\text { formato }\end{array}$ & Destinatario & Descripción \\
\hline $\begin{array}{l}\text { Checklist antes de } \\
\text { la prueba }\end{array}$ & $\begin{array}{l}\text { Cualquier miembro } \\
\text { del equipo }\end{array}$ & $\begin{array}{l}\text { Verificar que el lugar para la prueba este listo con lo necesario, que incluye } \\
\text { desde la instalación del software hasta la recepción de los usuarios. }\end{array}$ \\
$\begin{array}{l}\text { Checklist durante } \\
\text { las pruebas }\end{array}$ & Moderador & $\begin{array}{l}\text { Incluye el orden para realizar las actividades mostradas en el guión; el } \\
\text { último punto del guión será remplazado por agradecer la participación. }\end{array}$ \\
$\begin{array}{l}\text { Checklist después } \\
\text { de la prueba }\end{array}$ & $\begin{array}{l}\text { Todos los } \\
\text { miembros }\end{array}$ & $\begin{array}{l}\text { Al concluir la prueba se realizan varias actividades que van desde platicar } \\
\text { con los observadores para obtener sus opiniones, hasta recoger el material } \\
\text { usado. }\end{array}$ \\
\hline
\end{tabular}

\subsection{Ejecución de la prueba}

El día de la prueba, se debe tener a la mano todos los formatos en el orden que serán usados, llegar con anticipación al lugar de la evaluación para preparar lo necesario. Durante la prueba se debe seguir el guion o el checklist correspondiente. Al finalizar cada sesión o todas las pruebas platica con los observadores para recopilar sus observaciones, detectar problemas graves sobre la aplicación.

Sugerencias y/o recomendaciones:

1. Guiarse de los Checklist de la tabla 1. 
2. El moderador debe emplear tiempo antes de la prueba para platicar con el usuario con la finalidad de relajarlos y hacerlos sentir en confianza.

3. Evitar horarios usuales de comida, ya que los usuarios no estarán concentrados en la actividad; dependiendo del horario y del tiempo de la prueba se puede ofrecer dulces, agua, etc.

4. El equipo de evaluación debe ser amable con los usuarios, no sorprenderse o emitir sonidos cuando cometan errores los usuarios, evitar distracciones (teléfonos, visitas personales, platicar, usar zapatos que al pisar sean ruidosos, etc.).

5. Al finalizar las pruebas invierte tiempo con los usuarios, puesto que ellos en ocasiones proveen más información.

6. Reúnete con tu equipo de evaluación para hablar de mejoras en las pruebas.

\subsection{Análisis y reporte}

Al finalizar las pruebas se debe hacer un reporte, incluyendo lo siguiente: explicar brevemente la aplicación a evaluar, describir la planeación siguiendo el punto 3.1, describir el proceso que se llevó en la ejecución de la prueba, finalizar con el análisis del resultado.

El análisis de los resultados obtenidos incluye el total de los usuarios, cuántos son novatos, intermedios y expertos. Los resultados relacionados con la efectividad, eficiencia y satisfacción se representa usando tablas, gráficos, los valores mínimos y máximos, porcentaje o el número de éxitos del total de usuarios. La efectividad se resume al decir cuántos usuarios completaron cada tarea, mientras que la eficiencia se reporta cuántos usuarios lograron terminar cada una de las tareas en el tiempo esperado o fuera de tiempo, por ejemplo en la Tabla 2. Por otro lado, las herramientas para conocer la satisfacción proveen un resultado al finalizar la evaluación; en ese caso se puede poner los resultados de cada usuario y el total.

Recomendación y/o sugerencia:

1. referenciar a los usuarios por el número de participante no por el nombre.

2. Incluir los parámetros aceptables para la herramienta que mide la satisfacción, para que los lectores vean los valores de referencia usando dicha herramienta.

3. Usar los valores máximos y mínimos cuando el promedio no provea suficiente información.

4. La desviación estándar se sugiere cuando se tienen más de 10 usuarios.

Tabla 2. Ejemplo de resumir la eficiencia

\begin{tabular}{lccl}
\hline Actividad & \multicolumn{2}{c}{$\begin{array}{c}\text { Usuarios que } \\
\text { terminaron la actividad }\end{array}$} & Problemas encontrados \\
\cline { 2 - 3 } & $\begin{array}{l}\text { Dentro de } \\
\text { tiempo }\end{array}$ & $\begin{array}{c}\text { Fuera de } \\
\text { tiempo }\end{array}$ \\
\hline $\begin{array}{l}\text { 1.Registrarse en la } \\
\text { aplicación }\end{array}$ & 5 & 0 & -Ninguno \\
$\begin{array}{l}\text { 2.Comprar un par de } \\
\text { jeans }\end{array}$ & 3 & 2 & $\begin{array}{l}\text {-Para un usuario no fue evidente el botón } \\
\text { de compras } \\
\text {-La aplicación marco error con el } \\
\text { usuario 3, quien no completo en tiempo }\end{array}$ \\
\hline
\end{tabular}

\section{Proceso de pruebas}

Se probó el proceso al evaluar las siguientes 4 aplicaciones Web con diferentes equipos de evaluación que estaban formados por dos personas cada uno. El equipo 1 evalúo un punto de venta para un auto-lavado; el equipo 2 valoró una aplicación para que alumnos de carreras afines a la programación apliquen filtros sobre imágenes y accedan a ver el código aplicado. El equipo 3 evalúo una aplicación orientada a empresas que reclutan recursos humanos, y el equipo 4 evalúo una aplicación que permite ofrecer y solicitar servicios de reparación o limpieza para casas.

En general las evaluaciones ocurrieron sin problemas mayores, gracias a que siguieron las etapas mostradas en la Figura 1. Algunos de los problemas ocurridos y sus causas:

- El equipo 1 citó a sus 6 usuarios a la misma hora para evitar que llegaran tarde estos. Su plan era evaluar de dos en dos usuarios a la vez. El problema fue que tenían a todos los usuarios en el mismo cuarto, aun cuando este era espacioso; los usuarios sin actividad platicaban y distraían a quienes estaban concentrados evaluando la aplicación. Este equipo consideró que ellos dos eran suficiente personal para hacer la prueba, pero ellos se dieron cuenta de que no podían atender a los usuarios que esperaban ni a los que estaban haciendo la prueba a la vez.

- Tanto el equipo 2 como el 3 decidieron unirse para formar un equipo de evaluación de 4 integrantes, con la finalidad de evaluar a la vez a dos usuarios. En cada evaluación existían dos moderadores y dos observadores. El equipo 2 no tuvo problemas durante su ejecución de las pruebas; mientras el equipo 3 solo 
tuvo un problema debido a que los equipos utilizados no podían tener acceso a la red y su aplicación lo requería. Este evento hizo que se atrasaran las pruebas.

- El equipo 4 decidió evaluar un usuario a la vez, durante la ejecución de la prueba tuvieron tres problemas; uno de ellos fue que la cámara a usar no funcionó, la ventaja es que llevaban otra y se convirtió en un problema menor. El segundo problema fue que al momento de hacer la prueba se les ocurrió cambiar de roles, el que era observador cambio a moderador y viceversa; esto originó un poco de descontrol entre ellos. Otro de los problemas fue que no establecieron tiempo por cada tarea, una de ellas era registrarse en la aplicación, pero esta parte no era fácil de hacer y el usuario invirtió más tiempo del requerido, provocando que el usuario estuviera un poco nervioso.

\section{Conclusiones y trabajo a futuro}

Seguir un proceso para la evaluación de software permite tener más control y evitar imprevistos, más si los evaluadores son novatos. A partir de la propuesta, se observa que los evaluadores novatos al contar con un plan definido y los formatos de apoyo propuestos, les permiten actuar con mayor confianza durante todo el proceso. En el caso de que existan problemas técnicos (falla en la energía, en la red, etc.) se les sugiere mantener el control de la situación para que los usuarios en espera o en evaluación no se angustien. Este mismo proceso fue usado para hacer evaluaciones heurísticas, lo cual les permitió tener una mejor planeación. En dichas evaluaciones no se requiere usar observadores, ni registrar la satisfacción, la eficacia y la eficiencia, solo se requiere que los evaluadores expertos den sus opiniones de manera libre o siguiendo herramientas de evaluación.

Como trabajo futuro se planea aplicar la propuesta de evaluación en otros grupos de evaluadores novatos, con la finalidad de proveer más recomendaciones, así como detallar el proceso para evaluaciones heuristicas $\mathrm{y}$ considerar otras herramientas de evaluación.

\section{Agradecimientos.}

Este trabajo fue parcialmente apoyada por el Consejo Nacional de Ciencia y Tecnología (CONACyT) mediante el proyecto de Cátedras CONACyT "Infraestructura para Agilizar el Desarrollo de Sistemas Centrados en el Usuario" (Ref 3053).

\section{Referencias}

1. Miki, H.: Reconsidering the Notion of User Experience for Human-Centered Design. Human Interface and the Management of Information. Lecture Notes in Computer Science, Vol. 8016, pp. 329-337 (2013).

2. Preece, J.; Rogers Y.; Sharp H.: Interaction Design beyond HCI, Jhon Wiley\& Sons Inc. (Ed), pp. 389-460 (2002).

3. Lewis, J.: IBM computer usability satisfaction questionnaires: psychometric evaluation and instructions for use, International Journal of HCI, Vol. 7, pp. 57-78 (1995).

4. Rubin, J; Chisnell, D.: Handbook of usability testing: How to Plan, Design, and Conduct Effective Tests, Wiley Publishing, Inc. (Ed.), pp. 27-293 (2008).

5. Barnum, C.: Usability Testing Essentials Ready, Set...Test!. Elservier Inc. (Ed), pp. 83-312 (2011).

6. Nielsen J.; Landauer, T.: A Mathematical Model of the Finding of Usability Problems, Proceedings of ACM INTERCHI'93 Conference, pp. 206-213 (1993).

7. Hedlefs, M.; De la Garza, A.; Sanchez, M.; Garza, A.: Adaptación al español del cuestionario de usabilidad de sistemas informáticos CSUQ, Revista Iberoamericana de las Ciencias Computacionales e Informática, Vol. 4, No. 8, pp. 84-99 (2015).

8. QUIS (The Questionnaire for User Interaction Satisfaction), http://lap.umd.edu/quis/, Accedido el 4 de Julio de 2017.

9. SUMI, URL: http://sumi.uxp.ie/, Accedido: 4 de Julio de 2017.

10. Brooke, J.: SUS: a "quick and dirty" usability scale, Usability Evaluation in Industry, Taylor \& Francis (Ed), London, pp. 189-194 (1996).

11. Suárez; M.: PhD. SIRIUS: Sistema de Evaluación de la Usabilidad Web Orientado al Usuario y basado en la Determinación de Tareas Críticas, pp. 139-145 (2010). 